\title{
IS1245 restriction fragment length polymorphism typing of Mycobacterium avium from patients admitted to a reference hospital in Campinas, Brazil
}

A.C. Panunto,

M.C.B. Villares and M.C. Ramos
Faculdade de Ciências Médicas, Universidade Estadual de Campinas, Campinas, SP, Brasil

\section{Correspondence}

M.C. Ramos

Rua João Baptista Geraldi, 115

13085-852 Campinas, SP

Brasil

E-mail: mdecr@unicamp.br

Research supported by FAPESP

(No. 99/11332-5).

Received March 6, 2002

Accepted May 12, 2003

\begin{abstract}
Mycobacterium avium is an important pathogen among immunodeficient patients, especially patients with AIDS. The natural history of this disease is unclear. Several environmental sources have been implicated as the origin of this infection. Polyclonal infection with this species is observed, challenging the understanding of its pathogenesis and treatment. In the present study $45 \mathrm{M}$. avium strains were recovered from 39 patients admitted to a reference hospital between 1996 and 1998. Species identification was performed using a species-specific nucleic acid hybridization test (AccuProbe ${ }^{\circledR}$ ) from Gen-Probe ${ }^{\circledR}$. Strains were genotyped using IS1245 restriction fragment length polymorphism typing. Blood was the main source of the organism. In one patient with disseminated disease, $M$. avium could be recovered more than once from potentially sterile sites. Strains isolated from this patient had different genotypes, indicating that the infection was polyclonal. Four patient clones were characterized in this population, the largest clone being detected in eight patients. This finding points to a common-source transmission of the organism.
\end{abstract}

\section{Introduction}

Mycobacterium avium has emerged as a frequent pathogen among immunodeficient patients since the advent of AIDS (1). Although antiretroviral treatment has had a significant impact on the course of this disease (2), cases of $M$. avium infection still have a poor prognosis (3-5). The natural history of the disease has not been determined. It seems that the environment itself, including animal reservoirs more than hu- mans, represents the natural source of bacteria for these patients $(6,7)$. Disseminated disease is observed in AIDS patients, as evidenced by blood, bone marrow and deep tissue cultures (4,8-10). Polyclonal infection has been documented and seems to be frequent $(11,12)$.

Molecular typing techniques are important tools to determine the epidemiology of infectious diseases. Several methods based on amplification, hybridization or restriction can be applied to trace the mechanisms of 
disease. DNA fragment restriction and subsequent hybridization with IS1245 probes have been shown to be suitable for typing $M$. avium infections $(13,14)$.

The AIDS epidemic is a serious health problem. Infections caused by mycobacteria, mainly $M$. tuberculosis and $M$. avium, are frequently seen at our hospital. M. avium was identified in $45 \%$ of HIV-infected patients with mycobacterial diseases during the year 1996 (8).

The present study was designed to identify and characterize the genetic polymorphism of M. avium strains recovered from clinical specimens between 1996 and 1998.

\section{Material and Methods}

\section{Setting}

The study was performed at the Hospital de Clínicas, Universidade Estadual de Cam-

Table 1. Mycobacterium avium recovered from 39 patients during the 1996-1998 period.

\begin{tabular}{lc}
\hline Clinical specimen & Number of isolates (\%) \\
\hline Blood & $14(31.1)$ \\
Sputum & $12(26.7)$ \\
Stool & $5(11.1)$ \\
Urine & $4(8.9)$ \\
Liver biopsy & $3(6.7)$ \\
Bone marrow & $3(6.7)$ \\
Gastric lavage & $1(2.2)$ \\
Cerebrospinal fluid & $1(2.2)$ \\
Broncho-alveolar lavage & $1(2.2)$ \\
Skin & $1(2.2)$ \\
Total & 45
\end{tabular}

Table 2. Sources of clinical specimens, number of IS 1245 bands observed, and date of recovery of isolates from a patient with polyclonal and disseminated Mycobacterium avium infection.

\begin{tabular}{lcc}
\hline Source & $\begin{array}{c}\text { Number of } \\
\text { bands }\end{array}$ & $\begin{array}{c}\text { Date of } \\
\text { recovery }\end{array}$ \\
\hline Blood & 6 & $2 / 1 / 96$ \\
Blood & 17 & $9 / 1 / 96$ \\
Lung biopsy & 6 & $1 / 3 / 98$
\end{tabular}

pinas (HC-UNICAMP), Campinas, SP, Brazil. The hospital serves an area with an estimated population of 5 million people. All cultures positive for M. avium from 1996 through 1998 were analyzed.

\section{Isolation and identification of mycobacteria}

Primary isolation was performed under safety conditions in the Mycobacterial Laboratory. The isolates were cultivated in 12-B and 13-B bottles of the BACTEC 460TB radiometric culture system (Becton Dickinson $^{\circledR}$ Microbiology Systems, Sparks, MD, USA). Aliquots from positive bottles were transferred to Lowenstein-Jensen (LJ) medium. Mycobacteria were identified on fully grown LJ slants. Species identification was performed with the AccuProbe ${ }^{\circledR}$ M. avium complex Culture Identification Test (GenProbe ${ }^{\circledR}$ Incorporated, San Diego, CA, USA).

\section{Molecular strain typing}

IS1245 restriction fragment length polymorphism (RFLP) was determined according to an internationally standardized protocol (14). Fingerprints were analyzed visually and considered to be identical when all bands shared the same molecular weight positions. M. avium reference strain IWGMT49 with fragments of known molecular weights was analyzed together with unknown samples.

\section{Results}

Forty-five M. avium isolates were recovered from a variety of clinical specimens from 39 patients (Table 1). Almost all the patients included in the study $(\mathrm{N}=35)$ had documented HIV infection. Forty-three isolates could be typed with the IS1245 RFLP, while two strains were untypable because of the absence of detectable IS1245 copies. $M$. avium was recovered from blood, bone marrow, biopsy tissue or cerebrospinal fluid (CSF) in $47 \%$ of samples, with blood cul- 
tures being the main source of the organism.

The RFLP patterns of the 43 typable strains consisted of 6 to 23 bands with an average of 17 IS1245 copies per strain. For five patients, more than one isolate could be recovered. In one patient with disseminated disease, two strains with different genotypes were recovered from blood cultures drawn one week apart (Table 2), indicating that he had a polyclonal infection (fingerprints shown in Figure 1). Fingerprinting studies also showed that 16 of 39 patients (41\%) could be assigned to four IS1245 clusters. The individual clusters and the distribution of isolates according to the number of IS 1245 copies observed are shown in Figure 2. The largest cluster, arbitrarily designated MA18, consisted of eight patients. Some fingerprints of selected isolates belonging to this cluster are shown in Figure 3.

\section{Discussion}

Mycobacterium avium infections are frequently detected in AIDS patients in Brazil despite the wide use of highly active antiretroviral chemotherapy $(4,9)$. This infection has been associated with AIDS in the presence of the late immunosuppression that occurs when CD4+ cell counts are extremely low. Antiretroviral treatment has considerably improved the prognosis of AIDS by reducing the incidence of opportunistic events, but treatment failures, and non-adherence to therapy render individuals susceptible to many pathogens. In such patients M. avium has been isolated from many clinical sources, including stool, sputum, skin, blood, CSF, and biopsy tissues. Isolation of organisms from these body sites does not necessarily mean that they are a cause of morbidity, unless they are found at sterile sites concomitantly with clinical evidence such as anemia, weight loss and diarrhea $(4,9)$. The epidemiological events involved in the natural history of this infection have not yet been clarified.
Blood was the main source of M. avium in our study population, as also reported by others $(5,8)$, and confirms the disseminated character of this infection (4). Oplustil et al. (4) have shown that the M. avium complex was recovered from $58.4 \%$ of positive vials, corresponding to $53.6 \%$ of patients with positive blood cultures.

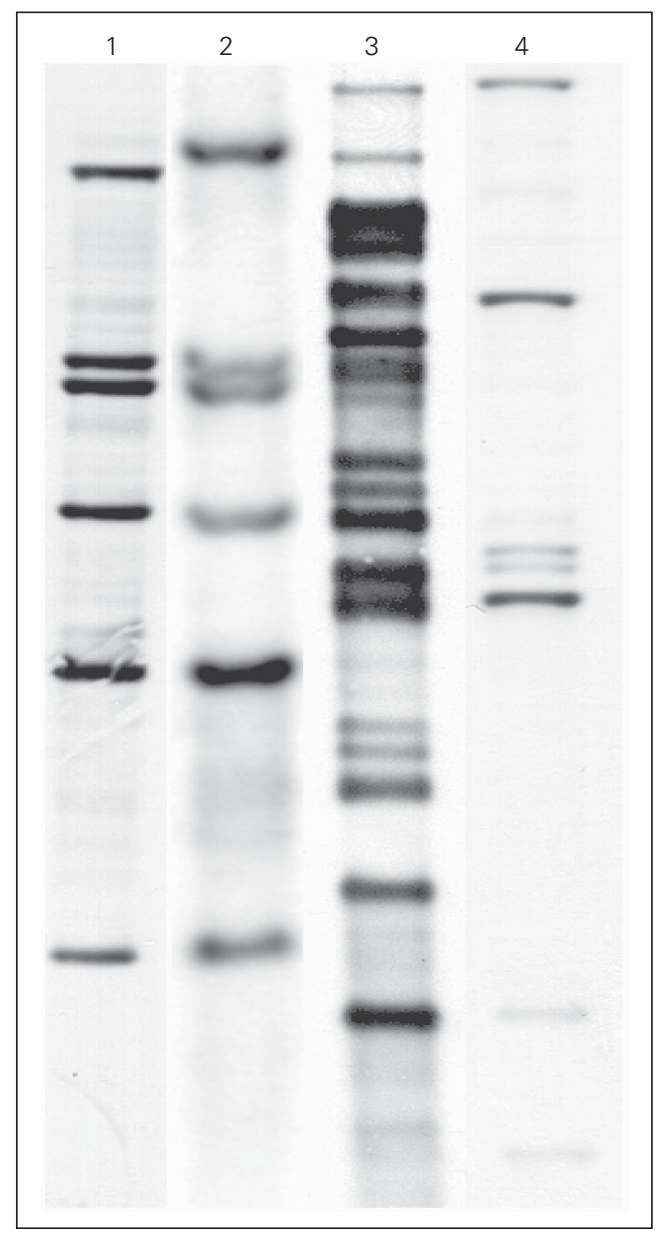

Figure 1. IS 1245 fingerprints of Mycobacterium avium isolated from a patient with multiple strains recovered. Lanes 1 and 2, Isolates from two blood samples; lane 3, isolate from lung tissue; lane 4, strain IWGMT49.

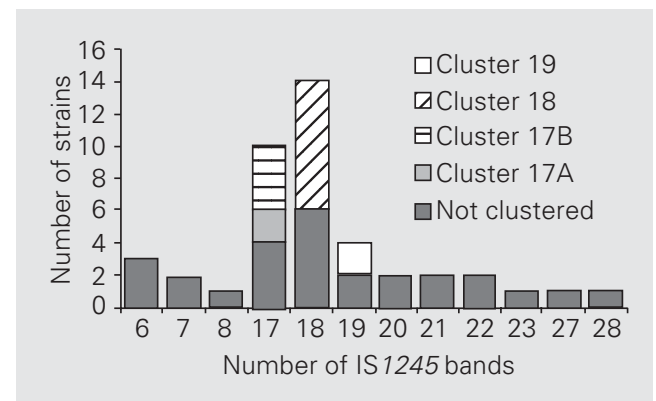

Figure 2. Number of isolates according to the number of IS 1245 bands observed. Clustered strains are outlined and represent one strain per patient. 
Figure 3. Some fingerprints from the largest cluster found, and arbitrarilly designated MA18.

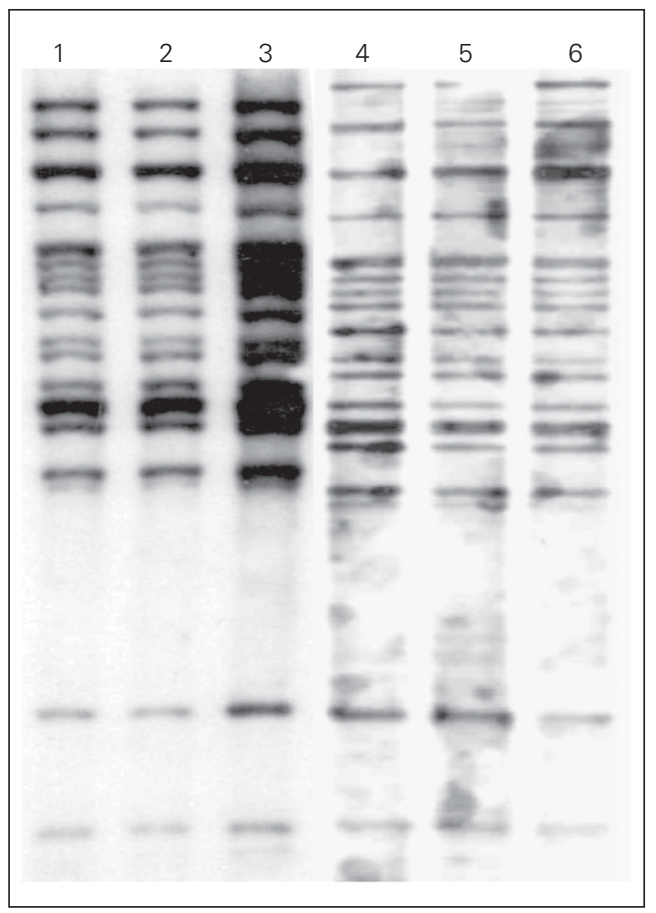

Our study also shows that $41 \%$ of the strains recovered from several clinical specimens were in clusters, indicating that these strains were acquired from common sources of infection. However, these sources of infection could not be determined in the present investigation. Since M. avium is not trans- mitted from human to human we suspect that the patients acquired the organism from the hospital environment, probably from water or food. In a recent study (15), it was concluded that $M$. avium complex infection results from diverse and probably undetectable environmental and nosocomial exposure.

Polyclonal infection was demonstrated in one patient in our study. This has been reported previously (12) and it has also been shown that organisms isolated from polyclonal infections demonstrated by organisms with different fingerprints can have different antimicrobial susceptibility (11).

Improved strategies to facilitate identification of HIV-infected people encourage patient enrollment in clinical care, and improve adherence to prevention. However, new treatment regimens will be needed before disseminated $M$. avium complex disease can be effectively prevented.

\section{Acknowlegements}

We thank Dr. Hanna Soini and Dr. Sylvia Leão for valuable suggestions and for supplying the $M$. avium reference strain IWGMT49.

\section{References}

1. Chaisson RE, Moore RD, Richman DD, Deruly J \& Creagh T (1992). The Zidovudine Epidemiology Study Group. Incidence and natural history of Mycobacterium avium-complex infection in patients with advanced human immunodeficiency virus disease treated with zidovudine. American Review of Respiratory Disease, 146: 285-289.

2. Rossi M, Flapp M, Telenti A et al. (2001). Disseminated M. avium complex infection in the Swiss HIV Cohort Study: declining incidence, improved prognosis and discontinuation of maintenance therapy. Swiss Medical Weekly, 131: 471-478.

3. Chaisson RE, Gallant JE, Keruly JC \& Moore RD (1998). Impact of opportunist disease on survival in patients with HIV infection. AIDS, 12: 29-33

4. Oplustil CP, Leite OH, Oliveira MS, Sinto SI, Uip DE, Boulos M \& Mendes CF (2001). Detection of mycobacteria in the bloodstream of patients with acquired immunodeficiency syndrome in a university hospital in Brazil. Brazilian Journal of Infectious Diseases, 5: 252-259.

5. Horsburgh Jr CR, Gettings J, Alexander LN \& Lennox JL (2001).
Disseminated Mycobacterium avium complex disease among patients infected with human immunodeficiency virus, 1985-2000. Clinical Infectious Diseases, 33: 1938-1943.

6. Leão SC, Briones MRS, Sircili MP, Balian SC, Mores N \& FerreiraNeto JS (1999). Identification of two novel Mycobacterium avium allelic variants in pig and human isolates from Brazil by PCR-restriction enzyme analysis. Journal of Clinical Microbiology, 37: 25922597.

7. Legrand E, Sola C \& Rastogi N (2000). Mycobacterium avium-intracellular complex: phenotypic and genotypic markers and the molecular basis for interspecies transmission. Bulletin de la Societé de Pathologie Exotique, 93: 182-192.

8. Ramos MC, Villares MCB, Jaques M, Roscani ALC, Roscani GN \& Alves EP (2000). Estudo bacteriológico retrospectivo das infecções micobacterianas em pacientes portadores da síndrome de imunodeficiência adquirida (SIDA). Brazilian Journal of Infectious Diseases, 4 : 120-126.

9. Hadad DJ, Palhares MC, Placco AL, Domingues CS, Castelo Filho A, 
Ferrazoli L, Ueki SY, Telles MA, Martins MC \& Palaci M (1995). Mycobacterium avium complex (MAC) isolated from AIDS patients and the criteria required for its implication in disease. Revista do Instituto de Medicina Tropical de São Paulo, 37: 375-383.

10. Sullivan AK, Hannan MM, Azadian BS, Easterbrook PJ, Gazzard BG \& Nelon MR (1999). Acid-alcohol fast bacilli in sputa of HIV-infected patients. International Journal of STD and AIDS, 10: 606-608.

11. Saad MHF, Telles MA, Porfirio F, Ferrazoli L, Fonseca LS, Johnson Jr W \& Riley LW (2000). Multiple isolates from AIDS patients: aspects of an analysis by a genotypic marker and antimicrobial susceptibility variations. Memórias do Instituto Oswaldo Cruz, 95: 729-732.

12. Oliveira RS, Sircili MP, Ueki SY, Telles MA, Schnabel B, Briones MR \& Leao SC (2000). PCR-restriction enzyme analysis of a bone mar- row isolate from a human immunodeficiency virus-positive patient discloses polyclonal infection with two Mycobacterium avium strains. Journal of Clinical Microbiology, 38: 4643-4645.

13. Saad MHF, Fonseca LS, Ferrazoli L et al. (1999). IS 1245 genotypic analysis of Mycobacterium avium isolates from AIDS patients in Brazil. International Journal of Infectious Diseases, 3: 192-196.

14. van Soolingen D, Bauer J, Ritacco V et al. (1998). IS 1245 restriction fragment length polymorphism typing of Mycobacterium avium isolates: proposal for standardization. Journal of Clinical Microbiology, 36: 3051-3054.

15. von Reyn CF, Arbeit RD, Horsburgh CR et al. (2002). Sources of disseminated Mycobacterium avium infection in AIDS. Journal of Infection, 44: 166-170. 